\title{
The significance of ultrasonographic carpal tunnel outlet measurements in the diagnosis of carpal tunnel syndrome
}

Anita Csillik MD ${ }^{1}$, Dániel Bereczki MD, PhD, DSc², László Bora $\mathrm{MD}^{3}$, Zsuzsanna Arányi MD, $\mathrm{PhD}^{1}$

${ }^{1}$ MTA-SE NAP B Peripheral Nervous System Research Group, Dept. of Neurology, Semmelweis University, Budapest, Hungary

${ }^{2}$ Dept. of Neurology, Semmelweis University, Budapest, Hungary

${ }^{3}$ Dept. of Radiology, Szent Lázár Regional Hospital, Salgótarján, Hungary

\section{Corresponding author:}

Anita Csillik MD

Dept. of Neurology, Semmelweis University

Address: Balassa u. 6, Budapest, Hungary-1083

E-mail: csillik.anita@med.semmelweis-univ.hu

Tel.: +36-20-825-0359, Fax: +36-1-210-1368

Conflicts of interest: None of the authors have potential conflicts of interest to be disclosed.

Acknowledgment: Anita Csillik and Zsuzsanna Arányi were supported by the Hungarian National Brain Research Program (NAP B) (KTIA_NAP_13-2-2014-0012). The sponsor had no direct involvement in the collection, analysis and interpretation of data and in the preparation of the manuscript. 


\section{HIGHLIGHTS}

- Median nerve enlargement in CTS is significantly greater at the tunnel outlet than at the inlet

- It is postulated that pressure progressively increases from proximal to distal within the tunnel

- The addition of outlet measurements increases diagnostic sensitivity and accuracy of CTS 


\section{ABSTRACT}

Objective: A retrospective study to investigate the utility of ultrasonographic carpal tunnel outlet measurements in the diagnosis of carpal tunnel syndrome (CTS).

Methods: 118 hands of 87 patients with electrophysiologically confirmed CTS and 44 control hands of 23 subjects were assessed. Cross-sectional areas (CSA) of the median nerve were measured at the tunnel inlet, outlet, and forearm. Longitudinal diameters (LAPD) were measured at the inlet, proximal tunnel, distal tunnel, and outlet.

Results: CSA at the outlet (median: $18 \mathrm{~mm}^{2}$ ) and its palm-to-forearm-ratio (median: 2.7) were significantly larger than CSA at the inlet (median: $15 \mathrm{~mm}^{2}$ ) and its wrist-to-forearm-ratio (median: 2.2) ( $\mathrm{p}<0.001$ ). $27 \%$ of the hands showed enlargement only at the outlet versus $13 \%$ only at the inlet. LAPD jump was significantly greater, suggesting relief of higher pressure, at the outlet / distal tunnel versus inlet / proximal tunnel $(\mathrm{p}<0.001)$.

Conclusion: Median nerve enlargement in CTS is greater at the tunnel outlet than at the inlet. We postulate that this is explained by the progressive increase of pressure within the tunnel from proximal to distal.

Significance: The addition of outlet measurements to inlet measurements increased sensitivity and accuracy of the ultrasonographic diagnosis of CTS by $15 \%$ and $10 \%$, respectively.

Key words: carpal tunnel syndrome; nerve ultrasound; carpal tunnel outlet; cross sectional area, palmto-forearm ratio 


\section{INTRODUCTION}

High resolution ultrasonography (HRUS) was first implemented as a diagnostic tool in carpal tunnel syndrome (CTS) in the early 1990s (Buchberger et al., 1991, 1992). The meta-analyses of the large body of literature that has accumulated in the past two decades (Cartwright et al., 2012; Tai et al., 2012) showed that level A evidence support the ultrasonographic measurement of median nerve crosssectional area at the tunnel inlet (CSA-I) as an accurate diagnostic test for CTS (Cartwright et al., 2012). The cut-off value of CSA-I showed a wide range of variation between 8 and $14 \mathrm{~mm}^{2}$, possibly due to issues of resolution, machine settings and individual examination technique, but most of the articles set the cut-off value of CSA-I at 9-11 mm² (Tai et al., 2012). Furthermore, with the idea of patients serving as their own control, the use of the wrist-to-forearm ratio (WFR) of CSA-I was also proposed (Hobson-Webb et al., 2008).

Although, the swelling of the median nerve (or generally any nerve under compression) may occur both proximally and distally to the compression, to date very little attention has been paid to measurements at the carpal tunnel outlet on the palm. Multilevel measurements have been advocated (Nakamichi et al., 2002; Wong et al., 2004; Chen et al., 2011), but the recent study of Paliwal et al. (2014) was the first to specifically address the utility of carpal tunnel outlet measurements. They concluded that the inclusion of median nerve measurements at the carpal tunnel outlet, in addition to inlet measurements, increased diagnostic sensitivity by $19 \%$. In line with these data, it was also our observation that in most CTS patients the swelling was conspicuous and often even more pronounced or isolated at the tunnel outlet on the palm than at the inlet at the wrist. Moreover, it was our impression that the degree of flattening of the median nerve within the carpal tunnel was usually the greatest in the distal part of the carpal tunnel under the distal edge of the retinaculum, and that the greater the distal flattening was, the greater was the swelling at the outlet. We have set out in the present paper to statistically analyse these observations with the aim of providing further evidence for the value of carpal tunnel outlet measurements in the diagnosis of CTS, and to test our hypothesis that the pronounced swelling at the outlet is related to increasing compression from proximal to distal in the tunnel. 


\section{PATIENTS AND METHODS}

Anonymised data were used retrospectively in accordance with the Helsinki Declaration. Approval for retrospective analysis of patient data was obtained from the Institutional Ethics Committee.

Between October 2014 and December 2015, 87 patients (118 hands) assessed by HRUS at the Department of Neurology, Semmelweis University, a tertiary referral centre for neuromuscular disorders, were included in this retrospective study. Inclusion criteria were the typical clinical symptoms and signs of idiopathic CTS and its electrophysiological confirmation. Clinical symptoms and signs included pain and paraesthesia exacerbated at night or provoked by a sustained wrist position, and relieved by changing position or shaking the hands; sensory deficit involving the median nerve distribution; and thenar atrophy. Patients ranged from mild (no clinical deficit) to extremely severe clinical symptoms (thenar atrophy, sensory loss). Exclusion criteria were bifid median nerve, previous CTS release on the relevant hand, and early division of the median nerve (within the canal or immediately at the outlet). Posttraumatic, pregnancy-related and other secondary CTS patients were excluded, but diabetes was not an exclusion criterion.

A control group was also examined, including 44 hands of 23 individuals. Control subjects were recruited from hospital staff and patients. None of the subjects had any clinical symptoms and signs suggestive for CTS. Subjects with diabetes or previous chemotherapy were excluded. As with patients, subjects with bifid median nerve, previous CTS release on the relevant hand, and early division of the median nerve were also excluded. Nerve conduction studies (NCS) were not performed on control subjects.

\subsection{Nerve conduction studies}

In 10 cases, electrophysiological testing was done at another institute. For the remaining 108 hands, NCS were performed at our institute using the Nicolet Viking Quest or EDX System (CareFusion Corporation) always by a physician trained in clinical neurophysiology, most often by two of the authors (A.C. and Z.A.). For confirmation of CTS, the following tests were done, as a standard 
protocol for CTS assessment: standard motor NCS with recording by surface electrodes from the abductor pollicis brevis muscle and stimulation at the wrist $(7 \mathrm{~cm}$ proximal to the active recording electrode), at the antecubital fossa and on the upper arm; standard antidromic sensory NCS with recording by ring electrodes over digit 2 and stimulation at the wrist and at the antecubital fossa; segmental antidromic sensory NCS to digit 2 with stimulation on the palm and at the wrist. The following NCS parameters were used for the diagnosis and grading of CTS: distal motor latency (DML), amplitude of the compound muscle action potential (CMAP) and of the sensory response (SNAP), the distal (digit-to-wrist) sensory conduction velocity, and the digit-to-palm and palm-towrist sensory conduction velocities and their difference. For comparison and to exclude generalised neuropathy, ulnar motor NCS to the abductor digiti minimi muscle, and antidromic sensory NCS to digit 5 with stimulation at the wrist were also performed. NCS reference values of our laboratory were used, with skin temperature controlled by the temperature probe of the EMG device (normal limits: DML $<4$ ms; distal sensory conduction velocity $>49$ m/s; CAMP $>4 \mathrm{mV}$; SNAP $>10 \mu \mathrm{V}$ ). Electrophysiological categories for the severity of CTS were set up, as defined in Table 1. The categories mild, moderate, moderately severe, severe, and extremely severe correspond to focal sensory demyelination, focal sensorimotor demyelination, focal sensorimotor demyelination with sensory axon loss, focal motor demyelination with sensorimotor axon loss, and complete sensorimotor denervation, respectively.

\subsection{Ultrasonography}

Ultrasonography was performed by two of the authors (A.C. and Z.A.), each with several years of experience in nerve ultrasound, with a Philips Epiq 5G ultrasound system and an 18-5 MHz linear array transducer. Settings were optimized for nerve imaging, including the use of compound imaging mode. Subjects were examined in supine position with the forearm in supinated and the fingers in neutral, semi-extended position. An effort was made to keep the transducer perpendicular to the median nerve to avoid anisotropy, which required tilting the transducer when scanning in the distal tunnel and at the outlet. CSA measurements were made by the continuous trace function of the ultrasound device within the inner border of the hyperechogenic epineural rim. The flexor retinaculum 
served as a landmark for the tunnel inlet and outlet: the tunnel inlet was defined as immediately proximal to the proximal edge of the retinaculum, and the tunnel outlet as immediately distal to the distal edge of the retinaculum; i.e. before the nerve enters and after the nerve leaves the tunnel, respectively. Scanning was started at the wrist in the axial plane relative to the nerve. At the tunnel inlet, approximately at the level of the distal wrist crease, the examiner repeatedly traced the nerve to and fro in order to identify the maximum nerve size where the CSA of the median nerve was measured (CSA-I). The nerve was then traced distally to the carpal tunnel outlet on the palm. The CSA measurement (CSA-O) was made where the flexor retinaculum was no longer visualized and the nerve was the largest before branching. Median nerve CSA was also measured at the forearm $12 \mathrm{~cm}$ proximal to the wrist crease. The WFR and the palm-to-forearm ratio (PFR) of the CSA values were calculated. To assess the degree of flattening of the nerve within the tunnel, longitudinal anteroposterior diameters (LAPDs) were used instead of the flattening ratio derived from the major and minor axis of the cross-section of the nerve, because intracarpal cross-sectional measurements are unreliable due to anisotropy. The whole length of the median nerve within the tunnel was scanned in the longitudinal plane and LAPDs were measured at four points: at the inlet as defined above (1), in the proximal carpal tunnel approximately at the level of the lunate-capitate border (2), in the distal carpal tunnel close to the distal edge of the flexor retinaculum (3), and at the outlet as defined above (4). LAPD measurements were made using the caliper function of the ultrasound device, between the inner borders of the hyperechogenic superficial and deep epineural sheath. With respect to the intracarpal proximal and distal LAPDs, measurements were made where the values were the smallest at these two levels.

In the control group, CSA and LAPD ultrasonographic measurements were made at the same sites as described for the patient group.

\subsection{Statistics}

Statistical analysis was performed by using Statistica for Windows v.12 program (StatSoft Tulsa OK. USA) with the significance level set at $\mathrm{p}<0.05$. Age, CSA-I, CSA-O, WFR, PFR and LAPD values 
were described by descriptive statistics. The Shapiro-Wilk W test was used to check normality.

Depending on normality, unpaired t-test or Mann-Whitney U test was used to compare age, CSA-I, CSA-O, WFR, PFR and LAPD values between the patient and the control groups. Paired t-test or Wilcoxon matched pairs signed rank test was used to perform CSA, WFR, PFR and LAPD comparisons within a group. The Kruskal Wallis test was used to compare WFR and PFR values among the different carpal tunnel severity groups. The cut-off values with the highest sensitivity and specificity for CSA-I, CSA-O, WFR and PFR were determined by receiver operating characteristic (ROC) analysis. The sensitivity, specificity, positive and negative predictive values, and accuracy of CSA-I, CSA-O, PFR and WFR and their combinations were calculated.

\section{RESULTS}

Eighty-seven patients (59 females and 28 males) with 118 affected hands (61 right and 57 left) were included in the analysis. The mean age of the patients was 65.8 years (SD: 15.5, range: 22-88).

Twenty-three individuals ( 15 females, 8 males) with 44 hands were enrolled as normal controls with a mean age of 60.0 years (SD: 15.5, range: 36-91). The difference between the age of the control and the patient groups was not significant $(\mathrm{p}=0.17$, Mann-Whitney $\mathrm{U}$ test).

Table 2 shows the median CSA values at the tunnel inlet and outlet, and their WFR and PFR ratios for both CTS patients and control subjects. The CSA of patients at both sites, and the WFR and PFR were significantly larger than those of the control group ( $\mathrm{p}<0.001$ for all comparisons, Mann-Whitney $\mathrm{U}$ test). In the patient group, the CSA at the tunnel outlet and the PFR were significantly larger than the CSA at the tunnel inlet and the WFR, respectively $(\mathrm{p}<0.001$ for both comparisons, Wilcoxon matched pairs signed rank test). Furthermore, 27\% of the hands showed CSA enlargement only at the outlet, and $13 \%$ only at the inlet. In the control group, no significant difference was found between CSA-I and CSA-O, and the PFR and WFR ( $\mathrm{p}=0.9$ for both comparisons, paired t-test). The optimal cut-off values for CSA-I, CSA-O, WFR, and PFR are shown in Table 3. Table 4 shows the sensitivity, specificity, positive and negative predictive values, and accuracy for CSA-I, CSA-O, WFR, PFR and 
their combinations. The sensitivity of CSA-O and of CSA-O and/or CSA-I were higher than that of CSA-I alone by $5.1 \%$ and $15.2 \%$, respectively. The sensitivity of WFR and PFR were both lower than their respective CSA measurements, but the sensitivity of PFR and of PFR and/or WFR was also higher than that of WFR alone by $10.2 \%$ and $20.4 \%$, respectively. Similarly, accuracy was higher by 9.9\% when both CSA-I and CSA-O were taken into account as opposed to CSA-I alone.

The nerve at the tunnel outlet typically appeared more hypoechogenic than at the inlet, but echogenicity was not quantified. Furthermore, anisotropy is more of an issue at the outlet because of the sloping in the course of the nerve in the dorsal direction.

LAPD measurements were made at four sites, as described in the Methods section. Table 5 shows the median LAPD values at these sites in the patient and the control groups. The point of maximum compression (flattening) within the tunnel was distal in all except for three hands (isolated proximal compression in one hand; combined proximal and distal compression with maximum proximal compression in one hand, and with equal degree of proximal and distal compression in one hand). A proximal notch sign (abrupt decrease of LAPD at the level of the lunate) was seen in further 15 hands, however with the exception of one hand compression was present also distally. The distal compression had several forms: 1 . an abrupt LAPD decrease, usually close to the distal of edge of the flexor retinaculum (33 hands); 2. continuously decreasing LAPD from proximal to distal (71 hands); 3. a distal LAPD drop in addition to the proximal compression (14 hands) (Figs 1-3). The LAPD in the distal carpal tunnel was significantly smaller than the LAPD in the proximal carpal tunnel (2 versus 3 : $\mathrm{p}<0.001$, Wilcoxon matched pairs signed rank test). In the control group, although the numerical difference between LAPD value 2 and 3 was very small, the difference proved to be statistically significant ( $\mathrm{p}<0.001$, Wilcoxon matched pairs signed rank test). The jump in LAPD between the tunnel inlet and the proximal tunnel (1-2) was $0 \%$ in the control and $23.1 \%$ in the patient group, whereas the jump in LAPD between the tunnel outlet and the distal tunnel (4-3) was $6.3 \%$ in the control and $127.3 \%$ in the patient group (median values). When statistically compared, within the patient group the jump in LAPD at the tunnel inlet (1-2) was significantly smaller versus the jump at the tunnel outlet (4-3) ( $\mathrm{p}<0.001$, Wilcoxon matched pairs signed rank test), whereas no such 
difference was found in the control group ( $\mathrm{p}=0.94$, Wilcoxon matched pairs signed rank test).

Furthermore, when the jump in LAPD at the tunnel inlet (1-2) and the outlet (4-3) in the patient group is compared to respective values in the control group, the difference was significant for both sites (p <0.001 for both comparisons, Mann-Whitney U test).

In 10 cases, where electrophysiological testing was done elsewhere, the electrophysiological severity was not determined. Among the remaining electrophysiologically classified 108 affected hands, 11 were assessed as extremely severe, 45 as severe, 16 as moderately severe, 28 as moderate, and 8 as mild. Neither the PFR, nor the WFR values showed a significant correlation with electrophysiological severity ( $\mathrm{p}=0.94$ and $\mathrm{p}=0.48$, respectively).

\section{DISCUSSION}

It has been shown experimentally that chronic nerve compression leads to endoneurial oedema, perineurial and endoneurial thickening and to other ultrastructural changes (Prinz et al., 2005), with consequent nerve enlargement. In CTS, the swelling of the nerve at the tunnel inlet has been very early recognized as a consistent sign of compression within the tunnel (Buchberger et al., 1991) and has become the mainstay for the ultrasonographic diagnosis of CTS (Cartwright et al., 2012; Tai et al., 2012). It has been postulated that the swelling resulting from compression is translated to the site of least resistance, which explains why it is seen at the proximal edge of the retinaculum, at the tunnel inlet, where the nerve is relieved from pressure, rather than within the confined space of the tunnel (Therimadasamy et al., 2012; Paliwal et al., 2014). On the other hand, such a relief and resulting swelling is expected at the distal edge of the retinaculum, at the tunnel outlet as well, an issue hitherto little addressed. We have shown in our cohort of idiopathic CTS patients that the median CSA at both the tunnel inlet and outlet is significantly larger than in the control group. Moreover, in CTS patients the CSA at the outlet and the PFR were significantly larger than the CSA at the inlet and the WFR, respectively, whereas no such difference was found in the control group. To note is that in $27 \%$ of the hands CSA enlargement was only seen at the outlet versus $13 \%$ only at the inlet. For the diagnosis of 
CTS, the CSA at the outlet was associated with higher values for all diagnostic indicators (sensitivity, specificity, positive and negative predictive values, and accuracy) than the CSA at the inlet (Table 4). When the CSA at the outlet and the inlet were both taken into account, sensitivity and accuracy increased by $15 \%$ and $10 \%$, respectively, as opposed to the CSA at the inlet alone (Table 4). The use of the derived ratios of WFR and PFR did not appear to increase diagnostic utility in our study. Altogether, these results confirm that swelling of the median nerve occurs at both ends of the tunnel in CTS and that swelling is even of greater degree at the tunnel outlet than at the inlet. Thus, the routine use of carpal tunnel outlet measurements is advocated.

Carpal tunnel outlet measurements were previously little addressed. This may be due to technical issues, as it may be difficult to trace the sloping hypoechogenic nerve distally on the palm, especially when resolution is lower. Moreover, the anatomical definition of the outlet varies among different studies. In previous studies reporting multilevel measurements (Nakamichi et al., 2002; Wong et al., 2004; Chen et al., 2011), the tunnel outlet and inlet have either not been clearly defined or defined as distal and proximal carpal tunnel. In our study, we have defined the tunnel inlet and outlet as the points immediately proximal to the proximal edge and distal to the distal edge of the flexor retinaculum, respectively. This is an important point as the greatest nerve swelling is to be expected where the nerve is just relieved from pressure. In the study of Nakamichi et al. (2002), three CSA measurements were made (proximal tunnel, mid-tunnel, distal tunnel) and concluded that the nerve shows marked intracarpal enlargement. This finding is difficult to interpret, considering that the nerve is under compression within the tunnel. Chen et al. (2011) performed an ultrasonographic 'inching test' with measuring CSA of the median nerve at 8 points along the carpal tunnel, and found that the greatest nerve enlargement was consistently seen at the most distal measuring sites on the palm. Although they have not defined these sites with respect to the distal edge of the flexor retinaculum, these distal sites most probably correspond to what we defined as the tunnel outlet, and thus their findings are closely in accordance with ours. More recently, the phenomenon of isolated nerve enlargement at the outlet in CTS was reported in a patient by Therimadasamy et al. (2012) and later by Paliwal et al. (2014). Similarly to our results, Paliwal et al. (2014) have also shown that the addition of 
CSA outlet measurement increased the sensitivity of ultrasound for the diagnosis of CTS, but they have not directly compared inlet and outlet measurements.

In our study, somewhat surprisingly electrophysiological severity did not show significant correlation with ultrasonographic inlet-outlet measurements. It may be related to the over-representation of hands with extremely severe and severe NCS findings in our CTS cohort. There is controversy in the literature concerning this issue. Some have found that CSA increased from mild to moderate severity (Chen et al., 2011), others have reported that ultrasonography can be complementary but not conclusive in the classification of CTS severities (Abrishamchi et al., 2015). On the other hand, in the study of Mhoon et al. (2012), no significant correlation was demonstrated between ultrasound parameters and electrophysiological severity and concluded that ultrasound cannot determine severity.

Our finding that nerve enlargement is greater at the outlet and that it is more often an isolated finding as opposed to the enlargement at the inlet was in parallel with the observation that nerve flattening, best seen on longitudinal images, usually appeared the greatest in the distal part of the tunnel. This finding was mentioned already in the first systematic description of CTS ultrasound signs (Buchberger et al., 1991). The point of maximum compression (flattening) within the tunnel, as indicated by the smallest LAPD, was distal in 115 out of the 118 hands examined, even if a notch sign was present at the proximal edge of the retinaculum. LAPD was significantly smaller in the distal than in the proximal carpal tunnel. Some degree of normal nerve flattening occurs from proximal to distal in the carpal tunnel (Bianchi and Martinoli, 2007), and there was a statistically significant difference in the control group as well, but the numeric difference was minor (Table 5). More importantly, a significantly greater jump of diameter at the border of outlet / distal tunnel versus inlet / proximal tunnel was demonstrated in the patient group, which suggests a relief of higher pressure at the outlet than at the inlet (in the control group there was no significant LAPD jump at either end, nor was there a difference between the two ends). Furthermore, in the majority of CTS hands continuously decreasing LAPD, i.e. progressive flattening was observed from proximal to distal, which indicates progressively increasing pressure and degree of compression within the tunnel from proximal to distal. It is an anatomical fact that the tunnel becomes progressively narrower from proximal to distal, and is 
the narrowest at the level of the hook of the hamate, at the distal insertion point of the flexor retinaculum (Rotman and Donovan, 2002; Bianchi and Martinoli, 2007). Moreover, it has been shown that the flexor retinaculum progressively thickens from proximal to distal, and it is the thickest distally and ulnarly, although there is also some thickening proximally and radially (Pacek et al., 2010; Goitz et al., 2014). These anatomical features suggest that the pressure within the tunnel should be higher in its distal portion. Indeed, it has been demonstrated with in vivo segmental carpal tunnel pressure measurements in patients with idiopathic CTS that the site of the highest pressure corresponded to the area around the hook of hamate (Murata et al., 2007), the narrowest part of the tunnel. Taken all these together, we postulate that the greater degree of nerve enlargement at the tunnel outlet as opposed to the tunnel inlet is a sign of greater degree of compression in the distal than in the proximal portion of the tunnel in most patients. This has clinical relevance as the location and the amount of flexor retinaculum that has to be released, especially in the distal portion, has been a controversial issue with the now widely used surgical method, the endoscopic carpal tunnel release (Murata et al., 2007). It has been reported that the incomplete release of the distal portion of the flexor retinaculum, including its hold-fast fibres (the fibrous structure between the distal flexor retinaculum and the palmar aponeurosis), may lead to incomplete alleviation of symptoms (Okutsu et al., 1996; Yoshida et al., 2007). We have also observed this on several occasions (Fig. 4). Thus, an isolated or marked median nerve enlargement at the tunnel outlet may serve as a 'whistleblower' for the surgeon to perform extensive distal carpal tunnel release.

Our study has limitations. The study was a retrospective analysis. Furthermore, because of the inability of making standard measurements, hands with bifid median nerve at the wrist or early branching within the tunnel were excluded from the analysis, resulting in some degree of selection bias and decreasing the power of our results. Nonetheless, as the majority of hands show standard anatomy, where standard inlet and outlet measurements are possible, our findings are relevant for the majority of patients. 


\section{CONCLUSIONS}

The results of our study indicate that median nerve enlargement occurs at both ends of the tunnel in carpal tunnel syndrome and that enlargement at the carpal tunnel outlet is even of greater degree and a more common isolated finding than enlargement at the inlet. We postulate that this is explained by the progressive increase of pressure within the tunnel from proximal to distal, related to the anatomy of the tunnel. We advocate the addition of carpal tunnel outlet measurements to increase the sensitivity and accuracy of the ultrasonographic diagnosis of CTS, and to serve as an indicator for surgeons for high distal pressure. 


\section{REFERENCES}

Abrishamchi F, Zaki B, Basiri K, Ghasemi M, Mohaghegh M. A comparison of the ultrasonographic median nerve cross-sectional area at the wrist and the wrist-to-forearm ratio in carpal tunnel syndrome. J Res Med Sci 2014; 19:1113-7.

Bianchi S, Martinoli C. In: Ultrasound of the Musculoskeletal System. Springer, 2007. pp: 446.

Buchberger W, Judmaier W, Birbamer G, Lener M, Schmidauer C. Carpal tunnel syndrome: diagnosis with high-resolution sonography. AJR Am J Roentgenol 1992; 159:793-8.

Buchberger W, Schoen G, Strasser K, Jungwirth W. Highresolution ultrasonography of the carpal tunnel. J Ultrasound Med 1991; 10:531-7.

Cartwright MS, Hobson-Webb LD, Boon AJ, Alter KE, Hunt CH, Flores VH et al. Evidence-based guideline: neuromuscular ultrasound for the diagnosis of carpal tunnel syndrome. Muscle Nerve 2012; 46:287-93.

Chen SF, Lu CH, Huang CR, Chuang YC, Tsai NW, Chang CC et al. Ultrasonographic median nerve cross-section areas measured by 8-point "inching test" for idiopathic carpal tunnel syndrome: a correlation of nerve conduction study severity and duration of clinical symptoms. BMC Med Imaging $2011 ; 11: 22$

Goitz RJ, Fowler JR, Li ZM. The Transverse Carpal Ligament: Anatomy and Clinical Implications. J Wrist Surg 2014; 3: 233-4.

Hobson-Webb LD, Massey JM, Juel VC, Sanders DB. The ultrasonographic wrist-to-forearm median nerve area ratio in carpal tunnel syndrome. Clin Neurophysiol 2008; 119:1353-57.

Mhoon JT, Juel VC, Hobson-Webb LD. Median nerve ultrasound as a screening tool in carpal tunnel syndrome: correlation of cross-sectional area measures with electrodiagnostic abnormality. Muscle Nerve 2012; 46:871-8. 
Murata K, Yajima H, Maegawa N, Hattori K, Takakura Y. Investigation of segmental carpal tunnel pressure in patients with idiopathic carpal tunnel syndrome--is it necessary to release the distal aponeurotic portion of the flexor retinaculum in endoscopic carpal tunnel release surgery? Hand Surg 2007; 12:205-9.

Nakamichi K-I, Tachibana S. Ultrasonographic measurement of median nerve cross-sectional area in idiopathic carpal tunnel syndrome: Diagnostic accuracy. Muscle Nerve 2002; 26:798-803.

Okutsu I, Hamanaka I, Tanabe T, Takatori Y, Ninomiya S. Complete endoscopic carpal canal decompression, Am J Ortop 1996; 25:365-8.

Pacek CA, ChakanM, Goitz RJ, Kaufmann RA, Li ZM. Morphological analysis of the transverse carpal ligament. Hand (NY) 2010; 5: 135-40.

Paliwal PR, Therimadasamy AK, Chan YC, Wilder-Smith EP. Does measuring the median nerve at the carpal tunnel outlet improve ultrasound CTS diagnosis? J Neurol Sci 2014; 339:47-51.

Prinz RA, Nakamura-Pereira M, De-Ary-Pires B, Fernandes D, Fabião-Gomes BD, Martinez AM et al. Axonal and extracellular matrix responses to experimental chronic nerve entrapment. Brain Res 2005; 1044:164-75.

Rotman MB, Donovan JP. Practical anatomy of the carpal tunnel. Hand Clin 2002; 18:219-30.

Tai TW, Wu CY, Su FC, Chern TC, Jou IM. Ultrasonography for diagnosing carpal tunnel syndrome: a meta-analysis of diagnostic test accuracy. Ultrasound Med Biol 2012; 38:1121-8.

Therimadasamy A, Pin Peng Y, Wilder-Smith EP. Carpal tunnel syndrome--median nerve enlargement restricted to the distal carpal tunnel. Muscle Nerve 2012; 46:455-7.

Wong SM, Griffith JF, Hui AC, Lo SK, Fu M, Wong KS. Carpal Tunnel Syndrome: Diagnostic Usefulness of Sonography 1. Radiology 2004; 232:93-9. 
Yoshida A, Okutsu I, Hamanaka I. Is complete release of all volar carpal canal structures necessary for complete decompression in endoscopic carpal tunnel release? J Hand Surg Eur Vol 2007; 32:537-42. 


\section{FIGURE LEGENDS}

\section{Figure 1}

\section{Carpal tunnel syndrome with a double notch sign}

The upper image shows the longitudinal scan of the median nerve with a proximal and a more pronounced distal notch sign. The lower images show the nerve in cross-section at the inlet (right) and the outlet (left). Note that the enlargement at the outlet is almost double than that at the inlet. The PFR is 3.7 and the WFR is 2.0 .

Dist: distal; Prox: proximal; Rad: radial; Uln: ulnar; Lun: lunate bone; Cap: capitate bone; MC: metacarpus; Trap: trapezium; CSA: cross-sectional area; PFR: palm-to-forearm ratio; WFR: wrist-to-forearm ratio

\section{Figure 2}

\section{Carpal tunnel syndrome with focal distal compression}

The upper image shows the longitudinal scan of the median nerve with a focal compression at the distal edge of the retinaculum. The lower images show the nerve in cross-section at the inlet (right) and the outlet (left). Note that the enlargement at the outlet is over double than that at the inlet. The CSA at the wrist is just over normal range. The PFR is 4.3 and the WFR is 2.1 .

Dist: distal; Prox: proximal; Rad: radial; Uln: ulnar; Lun: lunate bone; Cap: capitate bone; CSA: cross-sectional area; PFR: palm-to-forearm ratio; WFR: wrist-to-forearm ratio

\section{Figure 3}

\section{Carpal tunnel syndrome with compression gradually increasing distally}

The upper image shows the longitudinal scan of the median nerve with compression continuously increasing from proximal to distal. The lower images show the nerve in cross-section at the inlet (right) and the outlet (left). Note the marked difference in diameter at the outlet and the inlet. The PFR 
is 5.3 and the WFR is 1.8 .

Dist: distal; Prox: proximal; Rad: radial; Uln: ulnar; CSA: cross-sectional area; PFR: palm-to-forearm ratio; WFR: wrist-toforearm ratio

\section{Figure 4}

\section{Failed carpal tunnel surgery: incomplete release of the distal flexor retinaculum}

Symptoms of carpal tunnel syndrome failed to alleviate after endoscopic carpal tunnel release (patient not included in the study). Note the marked distal compression of the median nerve caused by the unsectioned distal part of the retinaculum, and the nerve enlargement at the outlet.

Dist: distal; Prox: proximal; Rad: radius; Lun: lunate bone; Cap: capitate bone 
Table 1

Electrophysiological criteria for severity categories in CTS

\begin{tabular}{|c|c|c|c|}
\hline Severity category & Segmental sensory NCS & Sensory NCS & Motor NCS \\
\hline Mild & palm-to-wrist NCV $\downarrow$ & normal & normal \\
\hline Moderate & palm-to-wrist NCV $\downarrow$ & digit-to-wrist NCV $\downarrow$ & DML norm. / mildly $\uparrow$ \\
\hline \multirow[t]{2}{*}{ Moderately severe } & palm-to-wrist NCV $\downarrow$ & digit-to-wrist NCV $\downarrow$ & $\mathrm{DML} \uparrow$ \\
\hline & SNAP ampl. $\downarrow$ & SNAP ampl. $\downarrow$ & \pm CMAP ampl. $\downarrow$ \\
\hline \multirow[t]{2}{*}{ Severe } & NR / SNAP ampl. $\downarrow$ on the & NR & $\mathrm{DML} \uparrow$ \\
\hline & palm & & CMAP ampl. $\downarrow$ \\
\hline Extremely severe & NR & NR & NR \\
\hline
\end{tabular}


Table 2

Median CSA-I, CSA-O, WFR and PFR values in the patient and control groups

\begin{tabular}{|c|c|c|c|c|c|c|}
\hline & $\begin{array}{c}\text { CSA-I mm }{ }^{2} \\
\text { (IQR) }\end{array}$ & $\begin{array}{c}\text { CSA-O mm } \\
\text { (IQR) }\end{array}$ & $\begin{array}{c}\text { CSA-I v. CSA-O } \\
\text { p value }\end{array}$ & $\begin{array}{l}\text { WFR } \\
\text { (IQR) }\end{array}$ & $\begin{array}{l}\text { PFR } \\
\text { (IQR) }\end{array}$ & $\begin{array}{c}\text { WFR v. PFR } \\
\text { p value }\end{array}$ \\
\hline CTS & $\begin{array}{c}15.0 \\
(13-18.1)\end{array}$ & $\begin{array}{c}18.0 \\
(14.4-21.8)\end{array}$ & $\mathrm{p}<0.001$ & $\begin{array}{c}2.2 \\
(1.9-3)\end{array}$ & $\begin{array}{c}2.7 \\
(2.2-3.3)\end{array}$ & $\mathrm{p}<0.001$ \\
\hline Control & $\begin{array}{c}10.1 \\
(9.2-10.8)\end{array}$ & $\begin{array}{c}10.1 \\
(8.6-12)\end{array}$ & $\mathrm{p}=0.9$ & $\begin{array}{c}1.5 \\
(1.4-1.7)\end{array}$ & $\begin{array}{c}1.6 \\
(1.4-1.8)\end{array}$ & $\mathrm{p}=0.9$ \\
\hline
\end{tabular}

Values are rounded to the nearest decimal. CTS: patients with carpal tunnel syndrome; CSA-I: cross-sectional area at the inlet; CSA-O: cross-sectional area at the outlet; WFR: wrist-to-forearm CSA ratio; PFR: palm-to-forearm CSA ratio; IQR: interquartile range 
Table 3

Optimal cut-off values for CSA-I, CSA-O, WFR and PFR

\begin{tabular}{|c|c|c|c|c|}
\hline & $\begin{array}{l}\text { CSA-I } \\
\left(\mathrm{mm}^{2}\right)\end{array}$ & $\begin{array}{l}\text { CSA-O } \\
\left(\mathrm{mm}^{2}\right)\end{array}$ & WFR & PFR \\
\hline Cut-off values & 12.6 & 13.2 & 2 & 2 \\
\hline Sensitivity & $80.4 \%$ & $86.2 \%$ & $68.2 \%$ & $80 \%$ \\
\hline Specificity & $87.1 \%$ & $93 \%$ & $89.5 \%$ & $89.6 \%$ \\
\hline AUC & 0.91 & 0.95 & 0.84 & 0.92 \\
\hline \multicolumn{5}{|c|}{ Cut-off, sensitivity and specificity values are rounded to the nearest decimal. } \\
\hline \multicolumn{5}{|c|}{ CSA-I: cross-sectional area at the inlet; CSA-O: cross-sectional area at the outlet } \\
\hline \multicolumn{5}{|c|}{ WFR: wrist-to-forearm CSA ratio; PFR: palm-to- forearm CSA ratio; } \\
\hline AUC: area under th & & & & \\
\hline
\end{tabular}




\section{Table 4}

Sensitivity, specificity, positive and negative predictive values and accuracy based on CSA enlargement at the outlet and inlet, abnormal WFR and PFR ratios, and their combinations

\begin{tabular}{|c|c|c|c|c|c|c|c|}
\hline & CSA-I & CSA-O & $\begin{array}{l}\text { CSA-I + } \\
\text { CSA-O }\end{array}$ & WFR & PFR & WFR + PFR & All four \\
\hline Sensitivity & $\begin{array}{c}79.7 \% \\
(94 / 118)\end{array}$ & $\begin{array}{c}\mathbf{8 4 . 8 \%} \\
(100 / 118)\end{array}$ & $\begin{array}{c}\mathbf{9 4 . 9 \%} \\
(112 / 118)\end{array}$ & $\begin{array}{l}\mathbf{6 8 . 6 \%} \\
(81 / 118)\end{array}$ & $\begin{array}{c}78.8 \% \\
(93 / 118)\end{array}$ & $\begin{array}{c}\mathbf{8 9 \%} \\
(105 / 118)\end{array}$ & $\begin{array}{c}96.6 \% \\
(114 / 118)\end{array}$ \\
\hline Specificity & $\begin{array}{l}86.4 \% \\
(38 / 48)\end{array}$ & $\begin{array}{l}93.2 \% \\
(41 / 44)\end{array}$ & $\begin{array}{l}81.8 \% \\
(36 / 44)\end{array}$ & $\begin{array}{l}90.9 \% \\
(40 / 44)\end{array}$ & $\begin{array}{l}90.9 \% \\
(40 / 44)\end{array}$ & $\begin{array}{l}81.8 \% \\
(36 / 44)\end{array}$ & $\begin{array}{l}70.5 \% \\
(31 / 44)\end{array}$ \\
\hline PPV & $\begin{array}{c}94 \% \\
(94 / 100)\end{array}$ & $\begin{array}{c}97.1 \% \\
(100 / 103)\end{array}$ & $\begin{array}{c}93.3 \% \\
(112 / 120)\end{array}$ & $\begin{array}{l}95.3 \% \\
(81 / 85)\end{array}$ & $\begin{array}{l}95.9 \% \\
(93 / 97)\end{array}$ & $\begin{array}{c}92.9 \% \\
(105 / 113)\end{array}$ & $\begin{array}{c}89.8 \% \\
(114 / 127)\end{array}$ \\
\hline NPV & $\begin{array}{l}61.3 \% \\
(38 / 62)\end{array}$ & $\begin{array}{l}69.52 \% \\
(41 / 59)\end{array}$ & $\begin{array}{l}85.7 \% \\
(36 / 42)\end{array}$ & $\begin{array}{r}52 \% \\
(40 / 77)\end{array}$ & $\begin{array}{l}61.5 \% \\
(40 / 65)\end{array}$ & $\begin{array}{l}73.5 \% \\
(36 / 49)\end{array}$ & $\begin{array}{l}88.6 \% \\
(31 / 35)\end{array}$ \\
\hline Accuracy & $\begin{array}{c}81.5 \% \\
([94+38] / 162)\end{array}$ & $\begin{array}{c}87 \% \\
([100+41] / 162)\end{array}$ & $\begin{array}{c}91.4 \% \\
([112+36] / 162)\end{array}$ & $\begin{array}{c}74.7 \% \\
([81+40] / 162)\end{array}$ & $\begin{array}{c}82.1 \% \\
([93+40] / 162)\end{array}$ & $\begin{array}{c}87 \% \\
([105+36] / 162)\end{array}$ & $\begin{array}{c}89.5 \% \\
([114+31 / 162)\end{array}$ \\
\hline
\end{tabular}

Values are rounded to the nearest decimal. CSA-I: cross-sectional area at the inlet; CSA-O: cross-sectional area at the outlet; WFR: wrist-to-forearm CSA ratio; PFR: palm-to- forearm CSA ratio; PPV: positive predictive value; NPV: negative predictive value; + : indicates and/or 


\section{Table 5}

Median LAPD values $(\mathrm{mm})$ in the patient and control groups

\begin{tabular}{lcc}
\hline Site of measurement & CTS & Control \\
& $($ IQR) & (IQR) \\
\hline (1) Tunnel inlet & 2.6 & 1.8 \\
& $(2.4-2.9)$ & $(1.7-2)$ \\
(2) Proximal carpal tunnel & 2 & 1.8 \\
(3) Distal carpal tunnel & $(1.8-2.2)$ & $(1.6-2)$ \\
& 1.1 & 1.6 \\
(4) Tunnel outlet & $(0.9-1.6)$ & $(1.5-1.9)$ \\
& 2.5 & 1.7 \\
& $(2.2-3)$ & $(1.5-1.9)$
\end{tabular}

Values are rounded to the nearest decimal. LAPD: longitudinal anteroposterior diameter;

CTS: carpal tunnel syndrome; IQR: interquartile range 


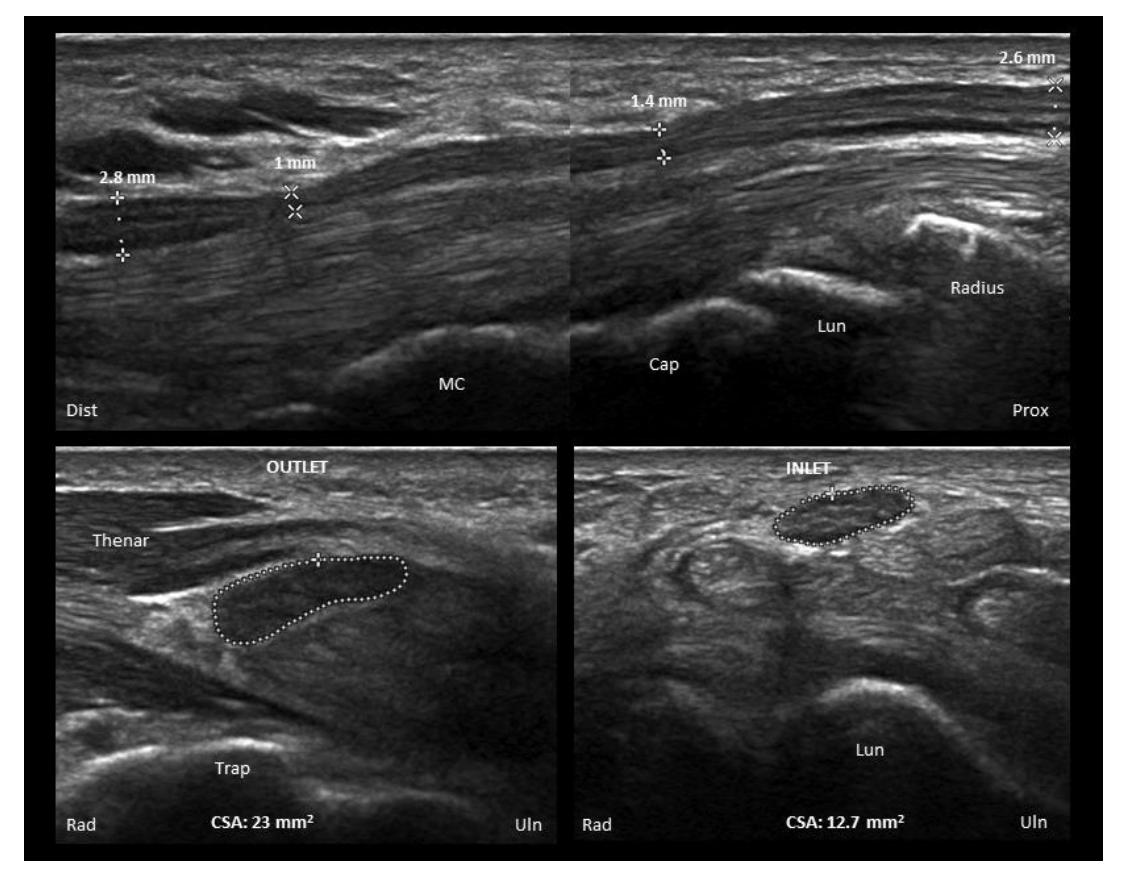

Fig. 1

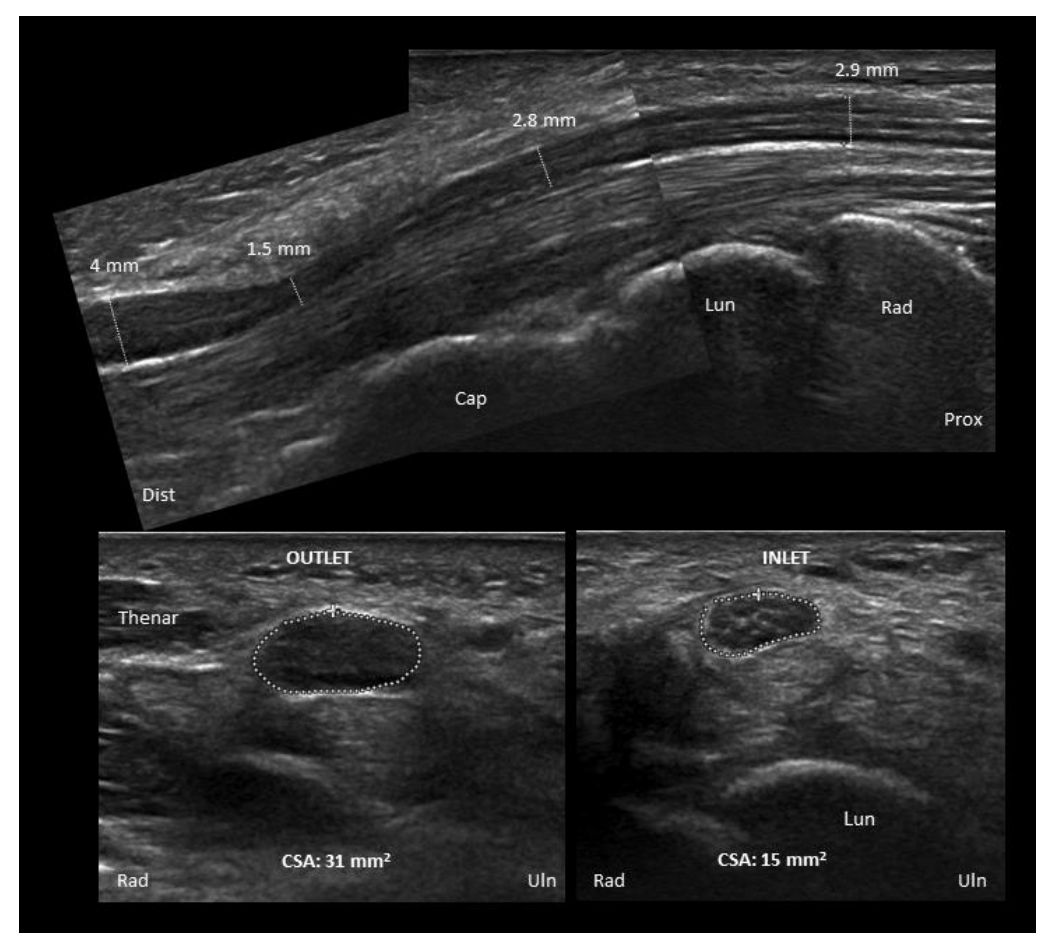

Fig. 2 


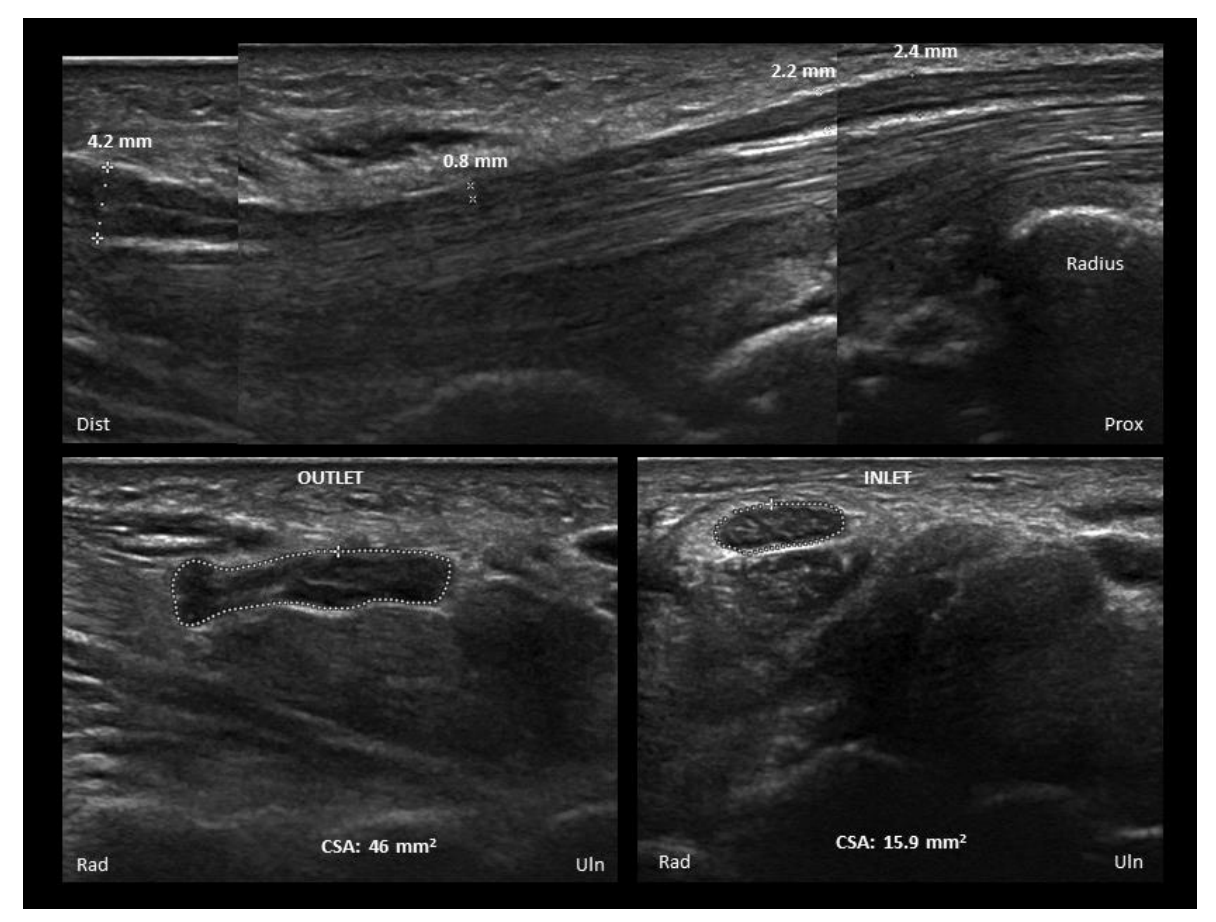

Fig. 3

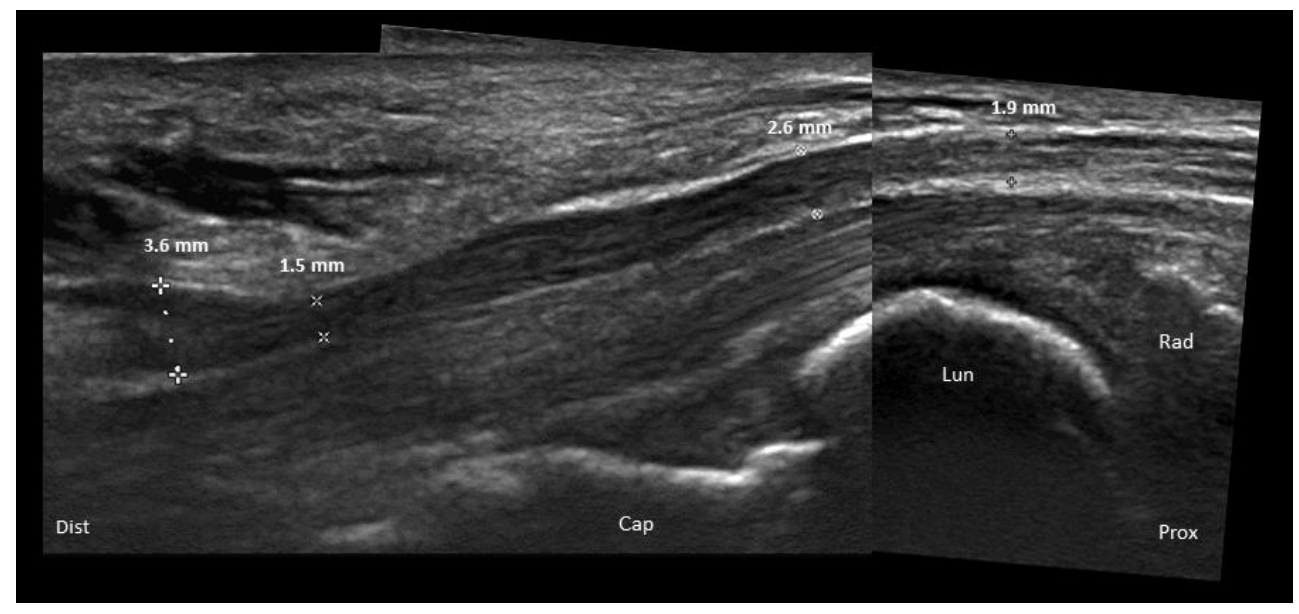

Fig. 4 